\title{
Adipokines, Insulin Resistance and Hyperandrogenemia in Obese Patients with Polycystic Ovary Syndrome: Cross-Sectional Correlations and the Effects of Weight Loss
}

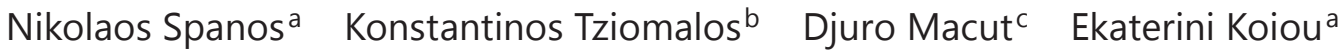 \\ Eleni A. Kandaraki ${ }^{a} \quad$ Dimitrios Delkos $^{a}$ Elena Tsourdi ${ }^{a}$ Dimitrios Panidis ${ }^{a}$ \\ a Division of Endocrinology and Human Reproduction, Second Department of Obstetrics and \\ Gynecology, Hippokration Hospital, b First Propedeutic Department of Internal Medicine, \\ AHEPA Hospital, Aristotle University of Thessaloniki, Thessaloniki, Greece, ' Institute of \\ Endocrinology, Diabetes and Metabolic Diseases, Clinical Centre of Serbia, Belgrade, Serbia
}

\section{Key Words}

Leptin · Adiponectin $\cdot$ Resistin $\cdot$ Visfatin $\cdot$ Polycystic ovary syndrome

\begin{abstract}
Objective: To assess the effects of weight loss on serum adipokine levels in polycystic ovary syndrome (PCOS). Methods: We determined serum leptin, adiponectin, resistin, and visfatin levels in 60 overweight/obese women with PCOS and 48 BMI-matched female volunteers. Measurements were repeated after 24 weeks of treatment with orlistat $120 \mathrm{mg} 3$ times per day along with an energy-restricted diet. Results: At baseline, serum visfatin concentration was higher in patients with PCOS than in controls ( $p=0.036)$; serum levels of leptin, adiponectin, and resistin did not differ between the two groups. After 24 weeks, a significant reduction in BMI and waist circumference was observed in both patients with PCOS and controls ( $p<0.001$ vs. baseline in both groups). Also serum leptin levels decreased in both patients with PCOS and controls ( $p<0.001$ vs. baseline in both groups). The reduction in serum leptin levels did not differ between groups. Serum adiponectin, resistin, and visfatin levels did not change in either group. Conclusions: Leptin, adiponectin, and resistin do not appear to play major pathogenetic roles in overweight/obese patients with PCOS. In contrast, visfatin emerges as a potentially important mediator of the endocrine abnormalities of these patients. However, serum visfatin levels are not substantially affected by weight loss.
\end{abstract}




\section{Introduction}

The polycystic ovary syndrome (PCOS) is a heterogeneous endocrine disorder with unclear pathogenesis $[1,2]$. Hyperandrogenism is a principal characteristic of the syndrome, and it has been suggested that increased androgen synthesis plays a major role in the pathogenesis of PCOS [1,2]. Insulin resistance (IR) is also frequently present in PCOS and appears to contribute to the increased steroidogenesis in these patients [1,2].

Obesity is a major risk factor for PCOS [1,2]. Visceral adipose tissue is a complex endocrine system that produces a large number of bioactive proteins, collectively termed adipokines [3]. The deregulated production of adipokines in obese subjects appears to be implicated in the pathogenesis of IR, hyperandrogenism, and PCOS [4,5]. Leptin, adiponectin, and resistin are among the most extensively studied adipokines $[3,6]$. Leptin is involved in body weight regulation, while adiponectin and resistin exert insulin-sensitizing and insulinantagonizing actions, respectively [3, 7]. Visfatin is a more recently described adipokine that also appears to have insulin-like effects $[3,7]$.

Several studies have assessed the role of specific adipokines in the pathogenesis of PCOS $[4,5]$. However, a comprehensive evaluation of the circulating levels of several adipokines in patients with PCOS has not been performed to date. In addition, there is a paucity of data on the effects of weight loss (i.e. the mainstay of treatment of overweight/obese patients with PCOS [1]) on serum adipokine levels in overweight/obese patients with PCOS [8-11]. Thus, the aim of the present study was to evaluate serum levels of leptin, adiponectin, resistin and visfatin in overweight/obese patients with PCOS, to determine their correlations with markers of hyperandrogenism, IR and obesity, and to assess the effects of weight loss on these levels. Based on previous studies [3,6-11], we hypothesized that weight loss would result in a decrease in serum leptin, resistin and visfatin levels and in an increase in serum adiponectin levels in both patients with PCOS and controls.

\section{Patients and Methods}

Patients

We prospectively studied 60 overweight/obese women with PCOS (mean age $25.4 \pm 6.2$ years, mean BMI $34.9 \pm 5.9 \mathrm{~kg} / \mathrm{m}^{2}$ ) and $48 \mathrm{BMI}-$ matched overweight/obese female volunteers (mean age $30.6 \pm 6.3$ years, mean BMI $34.9 \pm 6.1 \mathrm{~kg} / \mathrm{m}^{2}$ ) with normal ovulating cycles (28 \pm 2 days, blood progesterone levels $>10 \mathrm{ng} / \mathrm{ml}$ in two consecutive cycles), no signs of hyperandrogenism, and normal ultrasonographic appearance of the ovaries (table 1). Patients and controls were recruited between January 2008 and December 2009.

All women with PCOS were outpatients at the Gynecological Endocrinology Infirmary of the Second Department of Obstetrics and Gynecology, Aristotle University of Thessaloniki, Greece, who were diagnosed with PCOS according to the criteria that were proposed in 1990 [12] and revised in 2003 [13]. Among the patients with PCOS, 17, 26, 5 and 12 had phenotype 1, 2, 3 and 4, respectively [1]. Control women were healthy female members of the staff of our Department who volunteered to participate in the study.

None of the women studied had galactorrhea or any systematic disease that could possibly affect their reproductive physiology. No woman reported use of any medication during the last semester that could interfere with the normal function of the hypothalamic-pituitary-gonadal axis. When basic $17 \alpha$-hydroxyprogesterone ( $17 \alpha-\mathrm{OHP})$ levels were $>1.5 \mathrm{ng} / \mathrm{ml}$, the Synacthen test $(0.25 \mathrm{mg} / \mathrm{ml}$; Novartis Pharma S.A., Rueil-Malmaison, France) was performed to rule out congenital adrenal hyperplasia. Other causes of hyperandrogenemia, including prolactinoma, Cushing's syndrome and androgen-secreting tumors, were also excluded. Informed consent was obtained from all women, and the study was approved by the institutional review board. 
Spanos et al.: Adipokines, Insulin Resistance and Hyperandrogenemia in Obese Patients with Polycystic Ovary Syndrome: Cross-Sectional Correlations and the Effects of Weight Loss

Table 1. Baseline characteristics of patients with PCOS and controls

\begin{tabular}{|c|c|c|c|}
\hline & $\begin{array}{l}\text { Patients with PCOS } \\
(n=60)\end{array}$ & $\begin{array}{l}\text { Controls } \\
(n=48)\end{array}$ & $\begin{array}{l}\mathrm{p} \\
\text { (adjusted for age) }\end{array}$ \\
\hline Age, years & $25.4 \pm 6.2$ & $30.6 \pm 6.3$ & NA \\
\hline BMI, $\mathrm{kg} / \mathrm{m}^{2}$ & $34.9 \pm 5.9$ & $34.9 \pm 6.1$ & NS \\
\hline Waist, cm & $100.4 \pm 12.9$ & $99.0 \pm 12.8$ & NS \\
\hline $\mathrm{FSH}, \mathrm{mIU} / \mathrm{ml}$ & $5.9 \pm 1.7$ & $5.8 \pm 1.9$ & NS \\
\hline $\mathrm{LH}, \mathrm{mIU} / \mathrm{ml}$ & $7.4 \pm 5.3$ & $4.4 \pm 1.9$ & $<0.001$ \\
\hline Prolactin, ng/ml & $13.5 \pm 7.8$ & $12.1 \pm 5.1$ & NS \\
\hline Testosterone, ng/dl & $75.5 \pm 29.1$ & $48.7 \pm 18.3$ & $<0.001$ \\
\hline$\Delta 4-\mathrm{A}, \mathrm{ng} / \mathrm{ml}$ & $2.6 \pm 0.9$ & $1.7 \pm 0.6$ & $<0.001$ \\
\hline DHEA-S, ng/ml & $2,636.7 \pm 1362.9$ & $2,301.5 \pm 859.4$ & NS \\
\hline FAI & $10.63 \pm 6.29$ & $5.26 \pm 3.68$ & $<0.001$ \\
\hline $17 \alpha-\mathrm{OHP}, \mathrm{ng} / \mathrm{ml}$ & $1.1 \pm 0.5$ & $0.8 \pm 0.4$ & NS \\
\hline SHBG, nmol/l & $28.9 \pm 11.6$ & $42.5 \pm 26.5$ & 0.027 \\
\hline Glucose, mg/dl & $103.1 \pm 10.8$ & $104.8 \pm 10.8$ & NS \\
\hline Insulin, $\mu \mathrm{IU} / \mathrm{ml}$ & $18.7 \pm 11.7$ & $16.4 \pm 11.4$ & NS \\
\hline Glucose/insulin & $7.69 \pm 4.77$ & $9.28 \pm 5.37$ & NS \\
\hline HOMA-IR & $4.85 \pm 3.48$ & $4.29 \pm 3.07$ & NS \\
\hline QUICKI & $0.31 \pm 0.03$ & $0.32 \pm 0.03$ & NS \\
\hline Area under the OGTT curve & $16,325.7 \pm 3034.2$ & $17,056.2 \pm 3010.6$ & NS \\
\hline Mean ovarian volume, $\mathrm{cm}^{3}$ & $8.7 \pm 4.7$ & $6.5 \pm 3.3$ & 0.012 \\
\hline Mean number of ovarian follicles & $10.7 \pm 4.3$ & $7.3 \pm 2.7$ & $<0.001$ \\
\hline Leptin, ng/ml & $54.6 \pm 38.6$ & $54.0 \pm 44.8$ & NS \\
\hline Adiponectin, $\mu \mathrm{g} / \mathrm{ml}$ & $5.6 \pm 2.7$ & $5.7 \pm 2.7$ & NS \\
\hline Resistin, ng/ml & $6.3 \pm 2.3$ & $6.6 \pm 2.7$ & NS \\
\hline Visfatin, $\mathrm{ng} / \mathrm{ml}$ & $18.9 \pm 15.1$ & $14.2 \pm 9.7$ & 0.036 \\
\hline
\end{tabular}

NA = Not applicable; NS = not significant.

\section{Study Design}

At baseline, anthropometric, metabolic, hormonal, and ultrasonographic parameters as well as serum adipokine levels were compared between patients with PCOS and controls (see 'Clinical and Laboratory Investigations' below). All women with PCOS and all controls were then prescribed a normal-protein, energy-restricted diet (basal metabolic rate $600 \mathrm{kcal} /$ day, consisting of $50 \%$ from carbohydrates, $30 \%$ from fats (10\% saturated) and $20 \%$ from proteins) and orlistat (Xenical ${ }^{\circledR}$, Roche Hellas S.A., Greece; 120 mg 3 times per day before each meal) for 24 weeks. All baseline investigations were repeated at the end of treatment with orlistat and diet (i.e. at 24 weeks).

\section{Clinical and Laboratory Investigations}

In all women, body weight, height, and waist circumference (W) were recorded. The body weight was determined with an analog weight scale with subjects wearing light clothing. The height was measured barefoot with a stadiometer. The BMI was estimated as body weight (in $\mathrm{kg}$ ) divided by the square of the height (in $\mathrm{m}$ ). The $\mathrm{W}$ was measured at the level of the umbilicus.

Baseline blood samples were drawn after an overnight fast between the 3rd and 7th day of the menstrual cycle in controls and between 3 and 7 days after a spontaneous bleeding episode in patients with PCOS. We determined serum levels of follicle stimulating hormone (FSH), luteinizing hormone (LH), prolactin (PRL), testosterone $(\mathrm{T}), \Delta 4$-androstendione $(\Delta 4-\mathrm{A})$, dehydroepiandrosterone sulfate (DHEA-S), $17 \alpha$-OHP, sex hormone-binding globulin (SHBG), glucose, and insulin. Immediately after the baseline blood sampling, an oral glucose tolerance test (OGTT) was performed; $75 \mathrm{~g}$ of glucose were administered, and serum glucose levels were determined after 30, 60, 90 and $120 \mathrm{~min}$. Transvaginal ultrasonography was also performed on the same day, and the volume of each ovary and the number of follicles in each ovary were determined. 
Spanos et al.: Adipokines, Insulin Resistance and Hyperandrogenemia in Obese Patients with Polycystic Ovary Syndrome: Cross-Sectional Correlations and the Effects of Weight Loss

At the initial evaluation (baseline), the basal metabolic rate (in kcal/day) of all women was calculated and adjusted for moderate daily physical activity as follows: In women 18-30 years of age: $(0.0621 \times$ weight $(\mathrm{kg})+2.0357) \times 240 \times 1.3$ and in women $>31$ years of age: $(0.0342 \times$ weight $(\mathrm{kg})+3.5377) \times$ $240 \times 1.3[14]$.

\section{Laboratory Methods}

Serum levels of glucose, insulin, FSH, LH, PRL, T, $\Delta 4-\mathrm{A}, \mathrm{SHBG}$, and $17 \alpha$-OHP were determined as described previously [15]. Serum leptin, adiponectin, resistin, and visfatin levels were determined with enzyme-linked immunosorbent assays (leptin: Mercodia Leptin ELISA, Mercodia AB, Uppsala, Sweden; adiponectin: Mercodia Adiponectin ELISA, Mercodia AB; resistin: human resistin ELISA, BioVendor Laboratorni medicina a.s., Modrice, Czech Republic; visfatin: human visfatin ELISA kit, BioVision Research Products, Mountain View, CA, USA). The sensitivity of the assays was $50 \mathrm{pg} / \mathrm{ml}, 1.25 \mathrm{ng} / \mathrm{ml}, 33 \mathrm{pg} / \mathrm{ml}$ and $30 \mathrm{pg} / \mathrm{ml}$ for leptin, adiponectin, resistin and visfatin, respectively. The intra-assay coefficient of variation was 2.8 or $2.3 \%, 3.0$ or $3.0 \%, 2.8$ or $3.4 \%$ and 2.9 or $3.1 \%$ for low or high levels of leptin, adiponectin, resistin and visfatin, respectively. The inter-assay coefficient of variation was 4.5 or $5.2 \%, 5.3$ or $5.8 \%, 5.1$ or $6.9 \%$ and 4.8 or $5.5 \%$, for low or high levels of leptin, adiponectin, resistin and visfatin, respectively. Free androgen index (FAI) was calculated as T (nmol/l) $\times 100 /$ SHBG (nmol/l) [16]. The homeostasis model assessment of IR index (HOMA-IR) was calculated according to the following formula: fasting insulin $(\mathrm{mIU} / \mathrm{l}) \times$ glucose $(\mathrm{mg} / \mathrm{dl}) / 405$ [17]. The quantitative insulin sensitivity check index (QUICKI) was calculated with the formula 1 / (log fasting glucose $(\mathrm{mg} / \mathrm{dl})+\log$ fasting insulin (mIU/l)) [18].

\section{Transvaginal Ultrasonography}

Transvaginal ultrasonography was performed by an experienced operator in all women. Ovarian volume was calculated as follows: Ovarian volume $=(\pi / 6) \times$ ovarian length $\times$ ovarian height $\times$ ovarian width. Polycystic ovaries were diagnosed when $\geq 12$ follicles with a diameter of $2-9 \mathrm{~mm}$ were present in one or both ovaries, or when the ovarian volume was $>10 \mathrm{~cm}^{3}$.

\section{Statistical Analysis}

Data analysis was performed with the statistical package SPSS (version 17.0; SPSS Inc., Chicago, IL, USA). All tested parameters followed normal distribution as assessed with the Kolmogorov-Smirnov test and are reported as mean \pm SD.

Because women with PCOS were younger than controls, comparisons between groups at baseline were performed with analysis of covariance adjusting for age. At baseline, correlations between serum levels of each adipokine (leptin, adiponectin, resistin, and visfatin) and other parameters were assessed with Pearson's correlation. Parameters that were significantly correlated with serum levels of an adipokine according to Pearson's correlation were included in a stepwise linear regression analysis model to identify independent correlations with this adipokine.

Changes between baseline and end-of-treatment in the two groups were assessed with two-way repeated measures analysis of variance with the Holm-Sidak method for multiple comparison testing. Since there are no studies on the effects of weight loss on serum visfatin and resistin levels in patients with PCOS and only 4 short-term (1-8 weeks) studies on the effects of weight loss on serum leptin and adiponectin levels in this syndrome [8-11], a formal power analysis to calculate the necessary sample size to detect significant changes in adipokine levels after weight loss could not be performed.

In all cases, a p value $<0.05$ was considered significant.

\section{Results}

Baseline characteristics of patients with PCOS and controls are shown in table 1. Patients with PCOS had higher serum LH, T and $\Delta 4$-A levels and greater FAI than controls $(\mathrm{p}<0.001$ for all comparisons), whereas serum SHBG levels were lower in the former ( $p=0.027)$. Markers of IR did not differ between the two groups. Regarding adipokine levels, serum visfatin concentration was higher in patients with PCOS ( $p=0.036)$, whereas serum levels of leptin, adiponectin, and resistin did not differ between patients with PCOS and controls. 
Table 2. Significant correlations between serum adipokine levels and other parameters in patients with PCOS

\begin{tabular}{|c|c|c|c|c|c|}
\hline \multicolumn{3}{|l|}{ Univariate analysis } & \multicolumn{3}{|c|}{ Stepwise linear regression analysis } \\
\hline parameter & $\mathrm{r}$ & $\mathrm{p}$ & parameter & $\mathrm{b}$ & $\mathrm{p}$ \\
\hline \multicolumn{6}{|l|}{ Leptin } \\
\hline BMI & 0.513 & $<0.001$ & BMI & 0.519 & $<0.001$ \\
\hline Waist & 0.442 & $<0.001$ & $\begin{array}{l}\text { mean number of ovarian } \\
\text { follicles in the two ovaries }\end{array}$ & -0.269 & 0.016 \\
\hline Glucose/insulin & -0.325 & 0.011 & & & \\
\hline QUICKI & -0.302 & 0.019 & & & \\
\hline Mean number of ovarian & -0.257 & 0.047 & & & \\
\hline follicles in the two ovaries & & & & & \\
\hline \multicolumn{6}{|l|}{ Adiponectin } \\
\hline SHBG & 0.259 & 0.046 & & & \\
\hline \multicolumn{6}{|l|}{ Resistin } \\
\hline Visfatin & 0.284 & 0.028 & visfatin & 0.284 & 0.028 \\
\hline $17 \alpha-\mathrm{OHP}$ & 0.262 & 0.043 & & & \\
\hline \multicolumn{6}{|l|}{ Visfatin } \\
\hline BMI & 0.323 & 0.012 & BMI & 0.323 & 0.012 \\
\hline Resistin & 0.284 & 0.028 & & & \\
\hline Waist & 0.256 & 0.048 & & & \\
\hline
\end{tabular}

Baseline correlations between serum adipokine levels and other parameters in patients with PCOS are shown in table 2. In stepwise linear regression analysis, serum leptin levels correlated positively with the BMI and negatively with the mean number of follicles $(\mathrm{p}<$ 0.001 and $p=0.016$, respectively), serum resistin levels correlated positively with serum visfatin levels $(p=0.028)$, and serum visfatin levels correlated positively with the BMI $(p=$ 0.012). Serum adiponectin levels correlated in univariate analysis only with serum SHBG levels $(r=0.259, \mathrm{p}=0.046)$.

Baseline correlations between serum adipokine levels and other parameters in controls are shown in table 3. In stepwise linear regression analysis, serum leptin levels correlated positively with the BMI and serum visfatin levels $(\mathrm{p}<0.001$ and $\mathrm{p}=0.033$, respectively), serum adiponectin levels correlated positively with serum FSH levels and negatively with serum insulin levels ( $p=0.001$ and $p=0.021$, respectively), serum resistin levels correlated positively with serum glucose levels $(\mathrm{p}=0.013)$, and serum visfatin levels correlated positively with age and serum leptin levels and negatively with serum FSH levels $(p=0.015, p=$ 0.028 and $\mathrm{p}=0.011$, respectively).

Changes in patients with PCOS and controls after 24 weeks of diet and treatment with orlistat are shown in table 4. Significant reductions in BMI and W were observed in patients with PCOS and controls ( $p<0.001$ vs. baseline in both groups); these changes were comparable in the two groups. Serum T levels decreased more in patients with PCOS than in controls $(\mathrm{p}<0.001)$. The FAI also decreased more in patients with PCOS than in controls $(\mathrm{p}<0.001)$. However, both serum T levels and the FAI remained higher in patients with PCOS than in controls after weight loss $(64.2 \pm 24.0$ vs. $45.7 \pm 18.0 \mathrm{ng} / \mathrm{dl}(\mathrm{p}<0.001)$ and $7.35 \pm$ 4.34 vs. $3.96 \pm 2.80(\mathrm{p}=0.001)$, respectively). Both groups showed significant and comparable improvements in markers of IR. The mean ovarian volume and the mean number of ovarian follicles did not change in either group. Regarding the changes in adipokine levels, 
Table 3. Significant correlations between serum adipokine levels and other parameters in controls

\begin{tabular}{|c|c|c|c|c|c|}
\hline \multicolumn{3}{|l|}{ Univariate analysis } & \multicolumn{3}{|c|}{ Stepwise linear regression analysis } \\
\hline parameter & $\mathrm{r}$ & $\mathrm{p}$ & parameter & $\mathrm{b}$ & $\mathrm{p}$ \\
\hline \multicolumn{6}{|l|}{ Leptin } \\
\hline BMI & 0.534 & $<0.001$ & BMI & 0.472 & $<0.001$ \\
\hline Visfatin & 0.380 & 0.008 & visfatin & 0.270 & 0.033 \\
\hline Waist & 0.370 & 0.010 & & & \\
\hline Glucose/insulin & -0.339 & 0.018 & & & \\
\hline DHEA-S & -0.309 & 0.033 & & & \\
\hline \multicolumn{6}{|l|}{ Adiponectin } \\
\hline $\mathrm{FSH}$ & 0.474 & 0.001 & FSH & 0.439 & 0.001 \\
\hline Waist & -0.391 & 0.006 & insulin & -0.298 & 0.021 \\
\hline Visfatin & -0.362 & 0.012 & & & \\
\hline QUICKI & 0.354 & 0.014 & & & \\
\hline Insulin & -0.350 & 0.015 & & & \\
\hline HOMA-IR & -0.332 & 0.021 & & & \\
\hline Glucose/insulin & 0.308 & 0.033 & & & \\
\hline \multicolumn{6}{|l|}{ Resistin } \\
\hline Mean ovarian volume & 0.319 & 0.031 & glucose & 0.364 & 0.013 \\
\hline Glucose & 0.308 & 0.033 & & & \\
\hline$\Delta 4-\mathrm{A}$ & -0.286 & 0.049 & & & \\
\hline \multicolumn{6}{|l|}{ Visfatin } \\
\hline FSH & -0.380 & 0.008 & FSH & -0.337 & 0.011 \\
\hline Leptin & 0.380 & 0.008 & age & 0.312 & 0.015 \\
\hline Adiponectin & -0.362 & 0.012 & leptin & 0.288 & 0.028 \\
\hline Age & 0.298 & 0.040 & & & \\
\hline $\mathrm{W}$ & 0.298 & 0.040 & & & \\
\hline $17 \alpha-\mathrm{OHP}$ & -0.294 & 0.042 & & & \\
\hline
\end{tabular}

serum leptin levels decreased in both patients with PCOS and controls $(\mathrm{p}<0.001$ vs. baseline in both groups). This reduction in serum leptin levels did not differ between the two groups. Serum adiponectin, resistin, and visfatin levels did not change in either group.

\section{Discussion}

In the present study, serum visfatin levels were higher in overweight/obese patients with PCOS than in BMI-matched healthy females. Only 3 studies assessed serum visfatin levels in overweight/obese patients with PCOS and yielded conflicting results [19-21]. Dikmen et al. [19] and Tan et al. [21] reported higher serum visfatin levels in patients with PCOS ( $n=38$ and $n=8$, respectively). In contrast, Kowalska et al. [20] did not find any difference in serum visfatin levels between 47 overweight/obese patients with PCOS and 20 BMI-matched controls. In our study and in the study by Kowalska et al. [20], serum visfatin levels were independently correlated with the BMI. Patients with PCOS in our study had considerably higher BMI than the patients in the study by Kowalska et al. [20] (34.9 \pm 5.9 vs. $30.9 \pm 3.9 \mathrm{~kg} / \mathrm{m}^{2}$, respectively). Therefore, it is possible that the increase in visfatin levels in PCOS is more pronounced and becomes significantly different compared with controls only in patients with more severe obesity. On the other hand, a positive correlation 


Published online: July 23, 2012

Spanos et al.: Adipokines, Insulin Resistance and Hyperandrogenemia in Obese Patients with Polycystic Ovary Syndrome: Cross-Sectional Correlations and the Effects of Weight Loss

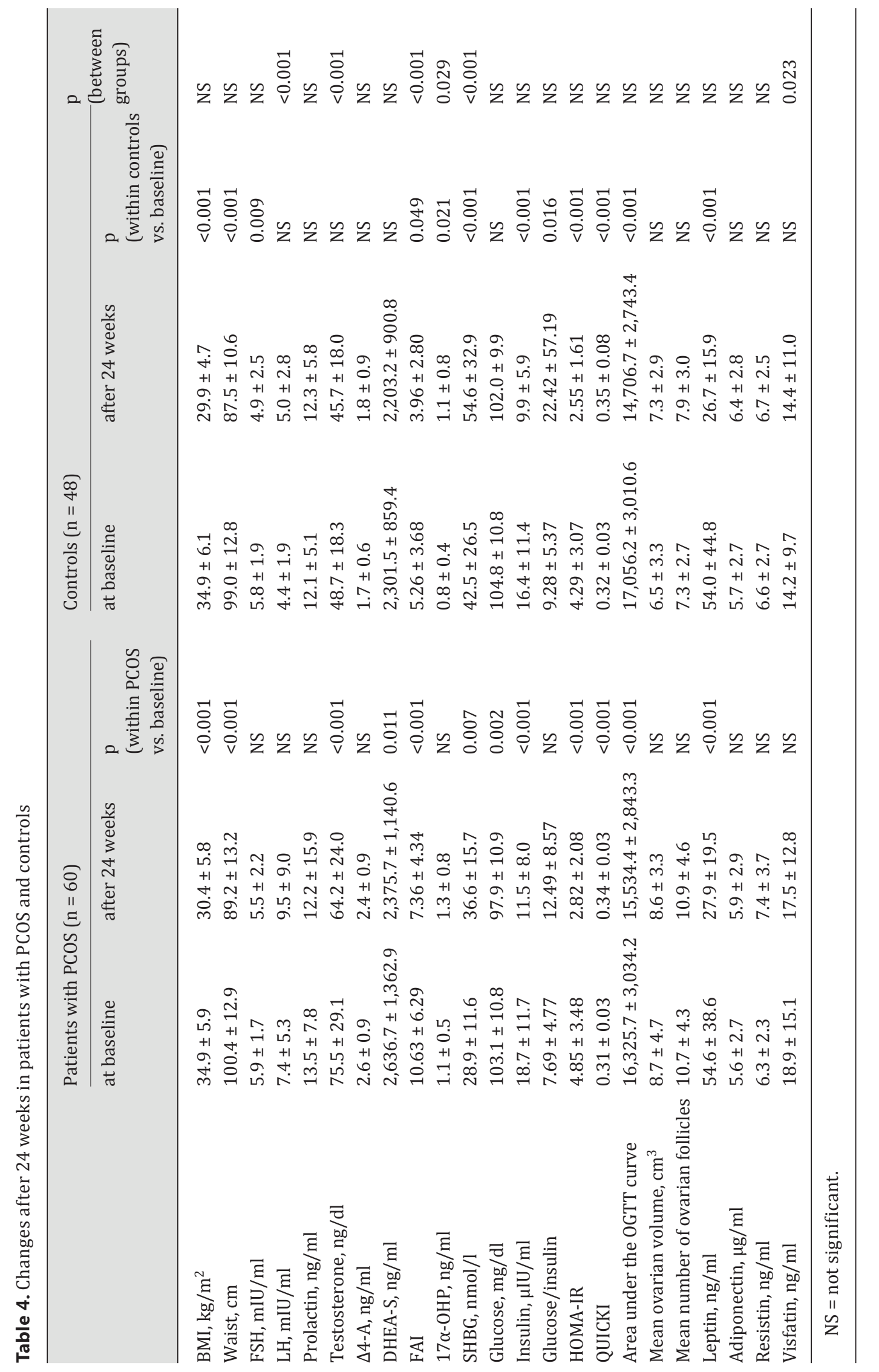


between serum visfatin levels and HOMA-IR was observed in the studies by Dikmen et al. [19] and Tan et al. [21]. We did not observe any association between serum visfatin levels and markers of IR, and neither did Kowalska et al. [20] who performed an euglycemic hyperinsulinemic clamp, i.e. the gold standard for assessing IR [22,23]. In addition, markers of IR did not differ between patients with PCOS and controls in our study. Therefore, IR does not appear to represent the major factor in the increase in serum visfatin levels in obese patients with PCOS. However, more studies are needed to clarify whether obesity, IR, or other abnormalities are primarily responsible for this increase.

Serum leptin, adiponectin, and resistin levels did not differ between patients with PCOS and controls in the present study; this finding is in agreement with previous reports [2430]. In addition, these adipokines did not correlate with any marker of IR, and this is also in accordance with the existing literature [24, 28-31]. Therefore, leptin, adiponectin, and resistin do not appear to play a major role in the pathogenesis of IR in PCOS.

In controls, we observed a significant negative correlation in stepwise linear regression analysis between serum adiponectin levels and serum insulin levels $(\mathrm{p}=0.021)$ as well as a significant positive correlation between serum resistin levels and serum glucose levels $(p=$ 0.013). These findings are in accordance with the insulin-sensitizing and -antagonizing action of adiponectin and resistin in normal subjects, respectively [3,7]. In contrast, neither of these correlations was observed in patients with PCOS, and this also supports the notion that the relations of these adipokines with insulin action are altered in PCOS. In addition, in controls, serum FSH levels correlated positively with serum adiponectin levels and negatively with serum visfatin levels ( $p=0.001$ and $p=0.011$, respectively). Again, neither of these correlations was observed in women with PCOS. Insulin appears to modulate the release of FSH from the pituitary [1]. Therefore, the correlation between FSH and both adiponectin and visfatin in controls might reflect the interaction of these adipokines with insulin signaling in healthy subjects. In contrast, the absence of similar correlations in women with PCOS also argues against a role for these adipokines in regulating insulin signaling in this disorder.

Weight loss resulted in a significant decline in serum leptin levels in both patients with PCOS and controls. Previous short-term (1-8 weeks) studies of the effects of hypocaloric diet in patients with PCOS also showed that serum leptin levels decline in parallel with weight loss $[8,10,11]$. This finding is somewhat expected because the degree of obesity is the major determinant of serum leptin levels in patients with PCOS [28-30]. Indeed, in agreement with previous reports, we observed a strong independent correlation between serum leptin levels and the BMI [28-30]. The close association between obesity and serum leptin levels is also suggested by our finding that a similar reduction in BMI in patients with PCOS and controls resulted in a comparable decrease in serum leptin levels in the two groups.

In contrast to the significant reduction in serum leptin levels, weight loss did not affect serum adiponectin, resistin, or visfatin levels. Previous 8-week studies that evaluated the effects of an energy-restricted diet on adiponectin levels in PCOS showed no change or a decrease in serum adiponectin levels $[8,9]$. On the other hand, the present study is the first that evaluated the impact of weight loss on serum resistin and visfatin levels in patients with PCOS. Our finding that serum adiponectin, resistin, or visfatin levels do not change in obese patients with PCOS despite a significant improvement in markers of IR argues against a role for these adipokines in the pathogenesis of IR in PCOS. It was somewhat unexpected that these adipokines did not change despite the substantial (approximately 12.9\%) weight loss. It is possible that longer follow-up is required for demonstrating significant changes in serum adiponectin, resistin, or visfatin levels after weight loss in patients with PCOS. However, there are no other studies that assessed the effects of weight loss on serum resistin 
Spanos et al:: Adipokines, Insulin Resistance and Hyperandrogenemia in Obese Patients with Polycystic Ovary Syndrome: Cross-Sectional Correlations and the Effects of Weight Loss

and visfatin levels in patients with PCOS, and more data are needed before reaching definite conclusions on the effects of diet on serum adipokine levels in PCOS. Of note, metformin reduced serum visfatin levels in one study in patients with PCOS and increased serum visfatin levels in another, whereas rosiglitazone and pioglitazone had no effect on serum visfatin levels [32-34]. However, these agents can simultaneously affect BMI and IR, and it is unclear which action contributes more to the change in serum visfatin levels [32-34]. Therefore, our findings on the effects of weight loss on serum adiponectin, resistin, and visfatin levels in PCOS remain to be confirmed or refuted by future studies.

Our study has some limitations. First, we studied only overweight/obese patients with PCOS and controls, and therefore our results might not apply to lean patients with PCOS. Second, IR was assessed with the HOMA-IR and QUICKI indices instead of the gold standard euglycemic hyperinsulinemic clamp [22,23]. However, the latter is cumbersome and difficult to apply repeatedly in large numbers of patients. Third, our study might had had limited power to detect significant changes in adipokine levels after weight loss and to discern differences in these changes between patients with PCOS and controls.

In conclusion, our study suggests that overweight/obese patients with PCOS have higher serum visfatin levels compared with BMI-matched controls. In contrast, serum leptin, adiponectin, and resistin levels do not differ between the two groups. Weight loss by diet and orlistat results in a significant reduction in serum leptin levels in both patients with PCOS and controls, and this decrease is comparable in the two groups. On the other hand, serum adiponectin, resistin, and visfatin levels are not affected by weight loss. Therefore, leptin, adiponectin, and resistin do not appear to play major pathogenetic roles in obese patients with PCOS while visfatin emerges as a potentially important mediator of the endocrine abnormalities of these patients. However, serum visfatin levels are not substantially affected by weight loss.

\section{Disclosure Statement}

We have no conflict of interest to declare.

\section{References}

1 Norman RJ, Dewailly D, Legro RS, Hickey TE: Polycystic ovary syndrome. Lancet 2007;370:685-697.

- 2 Matalliotakis I, Kourtis A, Koukoura O, Panidis D: Polycystic ovary syndrome: etiology and pathogenesis. Arch Gynecol Obstet 2006;274:187-197.

- 3 Rasouli N, Kern PA: Adipocytokines and the metabolic complications of obesity. J Clin Endocrinol Metab 2008;93(suppl 1):S64-S73.

- 4 Glintborg D, Andersen M: An update on the pathogenesis, inflammation, and metabolism in hirsutism and polycystic ovary syndrome. Gynecol Endocrinol 2010;26:281-296.

5 Garruti G, Depalo R, Vita MG, Lorusso F, Giampetruzzi F, Damato AB, Giorgino F: Adipose tissue, metabolic syndrome and polycystic ovary syndrome: from pathophysiology to treatment. Reprod Biomed Online 2009;19:552-563.

6 Trujillo ME, Scherer PE: Adipose tissue-derived factors: impact on health and disease. Endocr Rev 2006;27: 762-778.

7 Stofkova A: Resistin and visfatin: regulators of insulin sensitivity, inflammation and immunity. Endocr Regul 2010;44:25-36.

- 8 Kasim-Karakas SE, Almario RU, Cunningham W: Effects of protein versus simple sugar intake on weight loss in polycystic ovary syndrome (according to the National Institutes of Health criteria). Fertil Steril 2009;92:262-270.

9 Moran LJ, Noakes M, Clifton PM, Wittert GA, Belobrajdic DP, Norman RJ: C-reactive protein before and after weight loss in overweight women with and without polycystic ovary syndrome. J Clin Endocrinol Metab 2007;92:2944-2951. 
Spanos et al.: Adipokines, Insulin Resistance and Hyperandrogenemia in Obese Patients with Polycystic Ovary Syndrome: Cross-Sectional Correlations and the Effects of Weight Loss

10 Stamets K, Taylor DS, Kunselman A, Demers LM, Pelkman CL, Legro RS: A randomized trial of the effects of two types of short-term hypocaloric diets on weight loss in women with polycystic ovary syndrome. Fertil Steril 2004;81:630-637.

11 Van Dam EW, Roelfsema F, Veldhuis JD, Helmerhorst FM, Frolich M, Meinders AE, Krans HM, Pijl H: Increase in daily LH secretion in response to short-term calorie restriction in obese women with PCOS. Am J Physiol Endocrinol Metab 2002;282:E865-E872.

12 Zawadski JK, Dunaif A: Diagnostic criteria for polycystic ovary syndrome: towards a rational approach; in Dunaif A, Givens JR, Haseltine FP, Merriam GE, Hershman SM (eds): Polycystic Ovary Syndrome. Current Issues in Endocrinology and Metabolism. Boston, Blackwell, 1992, pp 377-384.

-13 Rotterdam ESHRE/ASRM-Sponsored PCOS Consensus Workshop Group: Revised 2003 consensus on diagnostic criteria and long-term health risks related to polycystic ovary syndrome. Fertil Steril 2004;81: 19-25.

14 Bray GA: Comtemporary Diagnosis and Management of Obesity. Newton, Handbooks in Health Care Co, 1998.

15 Piouka A, Farmakiotis D, Katsikis I, Macut D, Gerou S, Panidis D: Anti-Mullerian hormone levels reflect severity of PCOS but are negatively influenced by obesity: relationship with increased luteinizing hormone levels. Am J Physiol Endocrinol Metab 2009;296:E238-E243.

-16 Morley JE, Patrick P, Perry HM III: Evaluation of assays available to measure free testosterone. Metabolism 2002;51:554-559.

-17 Matthews DR, Hosker JP, Rudenski AS, Naylor BA, Treacher DF, Turner RC: Homeostasis model assessment: insulin resistance and beta-cell function from fasting plasma glucose and insulin concentrations in man. Diabetologia 1985;28:412-419.

18 Katz A, Nambi SS, Mather K, Baron AD, Follmann DA, Sullivan G, Quon MJ: Quantitative insulin sensitivity check index: a simple, accurate method for assessing insulin sensitivity in humans. J Clin Endocrinol Metab 2000;85:2402-2410.

19 Dikmen E, Tarkun I, Canturk Z, Cetinarslan B: Plasma visfatin level in women with polycystic ovary syndrome. Gynecol Endocrinol 2011;27:475-479..

-20 Kowalska I, Straczkowski M, Nikolajuk A, Adamska A, Karczewska-Kupczewska M, Otziomek E, Wolczynski $\mathrm{S}$, Gorska M: Serum visfatin in relation to insulin resistance and markers of hyperandrogenism in lean and obese women with polycystic ovary syndrome. Hum Reprod 2007;22:1824-1829.

-21 Tan BK, Chen J, Digby JE, Keay SD, Kennedy CR, Randeva HS: Increased visfatin messenger ribonucleic acid and protein levels in adipose tissue and adipocytes in women with polycystic ovary syndrome: parallel increase in plasma visfatin. J Clin Endocrinol Metab 2006;91:5022-5028.

22 DeFronzo RA, Tobin JD, Andres R: Glucose clamp technique: a method for quantifying insulin secretion and resistance. Am J Physiol 1979;237:E214-E223.

23 Diamanti-Kandarakis E, Kouli C, Alexandraki K, Spina G: Failure of mathematical indices to accurately assess insulin resistance in lean, overweight, or obese women with polycystic ovary syndrome. J Clin Endocrinol Metab 2004;89:1273-1276.

24 Carmina E, Orio F, Palomba S, Cascella T, Longo RA, Colao AM, Lombardi G, Lobo RA: Evidence for altered adipocyte function in polycystic ovary syndrome. Eur J Endocrinol 2005; 152:389-394.

25 Carmina E, Orio F, Palomba S, Longo RA, Cascella T, Colao A, Lombardi G, Rini GB, Lobo RA: Endothelial dysfunction in PCOS: role of obesity and adipose hormones. Am J Med 2006;119:356.

-26 Escobar-Morreale HF, Villuendas G, Botella-Carretero JI, Alvarez-Blasco F, Sanchon R, Luque-Ramirez M, San Millan JL: Adiponectin and resistin in PCOS: a clinical, biochemical and molecular genetic study. Hum Reprod 2006;21:2257-2265.

-27 Toulis KA, Goulis DG, Farmakiotis D, Georgopoulos NA, Katsikis I, Tarlatzis BC, Papadimas I, Panidis D: Adiponectin levels in women with polycystic ovary syndrome: a systematic review and a meta-analysis. Hum Reprod Update 2009;15:297-307.

28 Gennarelli G, Holte J, Wide L, Berne C, Lithell H: Is there a role for leptin in the endocrine and metabolic aberrations of polycystic ovary syndrome? Hum Reprod 1998;13:535-541.

29 Laughlin GA, Morales AJ, Yen SS: Serum leptin levels in women with polycystic ovary syndrome: the role of insulin resistance/hyperinsulinemia. J Clin Endocrinol Metab 1997;82:1692-1696.

-30 Mantzoros CS, Dunaif A, Flier JS: Leptin concentrations in the polycystic ovary syndrome. J Clin Endocrinol Metab 1997;82:1687-1691.

- 31 Panidis D, Kourtis A, Farmakiotis D, Mouslech T, Rousso D, Koliakos G: Serum adiponectin levels in women with polycystic ovary syndrome. Hum Reprod 2003;18:1790-1796.

-32 Aigner E, Bachofner N, Klein K, De GC, Hohla F, Patsch W, Datz C: Retinol-binding protein 4 in polycystic ovary syndrome - association with steroid hormones and response to pioglitazone treatment. J Clin Endocrinol Metab 2009;94:1229-1235.

-33 Ozkaya M, Cakal E, Ustun Y, Engin-Ustun Y: Effect of metformin on serum visfatin levels in patients with polycystic ovary syndrome. Fertil Steril 2010;93:880-884.

34 Steiner CA, Janez A, Jensterle M, Reisinger K, Forst T, Pfutzner A: Impact of treatment with rosiglitazone or metformin on biomarkers for insulin resistance and metabolic syndrome in patients with polycystic ovary syndrome. J Diabetes Sci Technol 2007;1:211-217. 